\title{
MicroRNA-18a-5p represses scar fibroblast proliferation and extracellular matrix deposition through regulating Smad2 expression
}

\author{
TIANSHI LI ${ }^{1}$, YIGUANG WU ${ }^{2}$, DANDAN LIU ${ }^{1}$ and LIDA ZHUANG ${ }^{1}$ \\ ${ }^{1}$ Department of Plastic and Cosmetic Surgery, Peking University Shenzhen Hospital, Shenzhen, Guangdong 518036; \\ ${ }^{2}$ Department of Food Science and Engineering, College of Chemistry and Environmental Engineering, \\ Shenzhen University Xili Campus, Shenzhen, Guangdong 518060, P.R. China
}

Received April 4, 2019; Accepted November 11, 2019

DOI: $10.3892 /$ etm.2021.10753

\begin{abstract}
The aim of the present study was to investigate the expression and role of microRNA-18a-5p (miR-18a-5p) during the formation of hypertrophic scar (HS), and to further explore the molecular mechanisms involved. Downregulation of miR-18a-5p in HS tissues and human HS fibroblasts (hHSFs) was detected by reverse transcription-quantitative polymerase chain reaction. The binding sites between miR-18a-5p and the 3 -untranslated region of SMAD family member $2(\operatorname{Smad} 2)$ were predicted by TargetScan and confirmed by dual-luciferase reporter assay. To investigate the role of miR-18a-5p in HS formation, the effects of miR-18a-5p downregulation or upregulation on hHSFs were subsequently determined. Cell proliferation was detected by an MTT assay, while cell apoptosis was measured by flow cytometry. In addition, the protein expression levels of Smad2, Collagen I (Col I) and Col III were examined by western blot assay. The findings indicated that miR-18a-5p downregulation in hHSFs significantly promoted the cell proliferation, decreased cell apoptosis and enhanced the expression levels of Smad2, Col I and Col III protein and mRNA, whereas miR-18a-5p upregulation in hHSFs exerted opposite effects. Notably, the effects of miR-18a-5p upregulation on hHSFs were eliminated by Smad2 upregulation. In conclusion, the data indicated that miR-18a-5p was downregulated during HS formation, and its upregulation repressed scar fibroblast proliferation and extracellular matrix deposition by targeting Smad2. Therefore, miR-18a-5p may serve as a novel therapeutic target for the treatment of HS.
\end{abstract}

Correspondence to: Dr Tianshi Li, Department of Plastic and Cosmetic Surgery, Peking University Shenzhen Hospital, 1120 Lianhua Road, Futian, Shenzhen, Guangdong 518036,P.R. China E-mail: litianshi0403@163.com

Key words: hypertrophic scar, microRNA-18a-5p, Smad2, hypertrophic scar fibroblasts, proliferation, extracellular matrix

\section{Introduction}

Hypertrophic scarring is a common complication of severe trauma, burns and surgical operation, resulting in dysfunction and deformity of the affected parts, which affects the quality of life of patients (1-3). In the formation and development of a hypertrophic scar (HS), the main features include the abnormal proliferation of fibroblasts and inhibition of apoptosis, as well as the imbalance of extracellular matrix collagen synthesis and degradation (4-7). Fibroblasts in HS tissue aggregate, and their number greatly increases. The proportion of Collagen I ( $\mathrm{Col} \mathrm{I})$ and Col III proteins (also known as type 1 and type 3 collagen, respectively) is abnormal and their content is increased during HS formation, which affects the scar appearance and even causes severe deformity and dysfunction. Currently, the main treatment for hypertrophic scar is surgical resection plus superficial X-ray radiation and dot-matrix laser. However, surgical treatment may cause secondary damage, and radiation therapy may cause radiation dermatitis, delayed healing of the incision and risk of skin cancer at the irradiated site (8). Therefore, it is of great significance to further understand the mechanism underlying the occurrence of HSs, and to identify new targets associated with the molecular mechanism for the prevention and treatment of these scars.

MicroRNAs (miRNAs) are endogenous, non-coding single-stranded small RNAs of approximately 22 nucleotides in length $(9,10)$. miRNAs can regulate gene expression through binding to the 3'-untranslated region (3'-UTR) of target mRNAs, and have emerged as key players in a wide array of biological processes, including cell proliferation and apoptosis (11-13). An increasing number of studies have reported that miRNAs are involved in the occurrence and development of HS (14-17). miR-18a-5p has been studied in several types of cancer, including osteosarcoma (18,19), glioma (20), breast cancer (21), prostate cancer (22), malignant melanoma (23), esophageal carcinoma (24), and renal cell carcinoma (25), among others. Furthermore, previous studies have revealed the inhibitory effect of miR-185-5p on cardiac fibrosis (26) and sub-pleural pulmonary fibrosis (27). However, to the best of our knowledge, the expression and functional role of miR-18a-5p in HS formation remain unclear. 
Therefore, the aim of the present study was to investigate the expression and role of miR-18a-5p in HS formation. Since fibroblast hyperplasia and extracellular matrix deposition are the main features of HS formation $(28,29)$, the effect of miR-18a-5p on scar fibroblast proliferation and extracellular matrix deposition was further investigated in the current study in order to explore the underlying mechanism by which miR-18a-5p is involved in HS formation.

\section{Materials and methods}

Clinical samples. A total of $40 \mathrm{HS}$ tissues from 40 patients who were subjected to scar excision (age range, 27-51 years old; gender ratio, 1:1) and 40 normal skin tissues from 40 patients subjected to auto-skin grafting (age range, 28-53 years old; gender ratio, 1:1) were collected at Peking University Shenzhen Hospital (Shenzhen, China) between February 2015 and February 2017. The tissues were immediately stored in liquid nitrogen until further use. For reverse transcription-quantitative PCR (RT-qPCR) analysis, the tissues were homogenized using an ultrasonic pulverizer (MSE Soniprep 150 Plus; MSE centrifuges). Written informed consent was obtained from each patient enrolled in the present study, and the study was approved by Human Ethical Committee of Peking University Shenzhen Hospital.

Cell culture. The human embryonic skin fibroblasts CCC-ESF-1 were obtained from Shanghai Zibo Biological Technology Co., Ltd. (cat. no. YB-ATCC-3084; Shanghai, China). Human HS fibroblasts (hHSFs) were provided by Shanghai Guandao Biological Engineering Co., Ltd. (cat. no. C0618; Shanghai, China). All cells were grown in Dulbecco's modified Eagle medium (DMEM; Invitrogen; Thermo Fisher Scientific, Inc., Waltham, MA, USA) containing $10 \%$ fetal bovine serum (Invitrogen; Thermo Fisher Scientific, Inc.) and incubated at $37^{\circ} \mathrm{C}$ with $5 \% \mathrm{CO}_{2}$.

Cell transfection. hHSFs were transfected with $100 \mathrm{nM}$ miR-18a-5p inhibitor (5'-CUAUCUGCACUAGAUGCACCU UA-3'; Guangzhou RiboBio Co., Ltd.), 100 nM inhibitor control (5'-UUCUCCGAACGUGUCACGUTT-3'; Guangzhou RiboBio Co., Ltd.), 100 nM miR-18a-5p mimic (sense, 5'-UAA GGUGCAUCUAGUGCAGAUAG-3' and anti-sense, 5'-AUC UGCACUAGAUGCACCUUAUU-3'; Guangzhou RiboBio Co., Ltd.), 100 nM mimic control (sense, 5'-UUCUCCGAA CGUGUCACGUTT-3' and anti-sense, 5'-ACGUGACAC GUUCGGAGAATT-3'; Guangzhou RiboBio Co., Ltd.), $1 \mu \mathrm{g}$ Smad2-plasmid (cat. no. sc-421525-ACT; Santa Cruz Biotechnology, Inc.), $1 \mu \mathrm{g}$ control-plasmid (cat. no. sc-108083; Santa Cruz Biotechnology, Inc.) or $100 \mathrm{nM}$ miR-18a-5p mimic $+1 \mu \mathrm{g} \mathrm{Smad} 2$-plasmid using Lipofectamine ${ }^{\circledR} 2000$ (Invitrogen; Thermo Fisher Scientific, Inc.) in line with the manufacturer's protocol. At $48 \mathrm{~h}$ after transfection, reverse transcriptionquantitative polymerase chain reaction (RT-qPCR) was performed to determine transfection efficiency.

RT-qPCR assay. In order to collect the total RNA from tissues or cells, TRIzol reagent (Invitrogen; Thermo Fisher Scientific, Inc.) was used in line with the manufacturer's protocol. RNA concentration was determined using a NanoDrop ${ }^{\mathrm{TM}}$ 2000c Spectrophotometer (Thermo Fisher Scientific, Inc.). For miRNA detection, reverse transcription and qPCR were performed using miScript II Reverse Transcription kit (Qiagen $\mathrm{GmbH}$ ) and miSCRIPT SYBR Green PCR Kit (Qiagen $\mathrm{GmbH}$ ), respectively, as per the manufacturer's protocols. For mRNA detection, PrimeScript ${ }^{\mathrm{TM}}$ RT reagent kit (Takara Bio, Inc.) was used for reverse transcription, followed by the SYBR Premix Ex Taq ${ }^{\mathrm{TM}}$ II (Tli RNaseH Plus) kit (Takara Bio, Inc.) was applied for qPCR analysis, following the manufacturer's protocol. The thermal cycling conditions were as follows: 35 cycles at $95^{\circ} \mathrm{C}$ for $15 \mathrm{sec}$, annealing at $60^{\circ} \mathrm{C}$ for $1 \mathrm{~min}$, then chain extension at $72^{\circ} \mathrm{C}$ for $1 \mathrm{~min}$ and a final extension step at $72^{\circ} \mathrm{C}$ for $10 \mathrm{~min}$. U6 and GAPDH were used as the internal controls for miRNA and mRNA, respectively. Primer sequences for PCR were listed as follows: GAPDH forward, 5'-TTTGGTATCGTGGAAGGACTC-3' and reverse, 5'-GTA GAGGCAGGGATGATGTTCT-3'; U6 forward, 5'-GCTTCG GCAGCACATATACTAAAAT-3' and reverse, 5'-CGCTTC ACGAATTTGCGTGTCAT-3'; miR-18a-5p forward, 5'-ACG TAAGGTGCATCTAGTGCAGATA-3' and reverse, 5'-GTG CAGGGTCCGAGGT-3'; type I collagen forward, 5'-CCC TGAGTGGAAGAGTGGAG-3' and reverse, 5'-GAGGCG TGAGGTCTTCTGTG-3'; Type III collagen forward, 5'-GGA GCTGGCTACTTCTCGC-3' and reverse, 5'-GGGAACATC CTCCTTCAACAG-3' and Smad2 forward, 5'-CGTCCATCT TGCCATTCACG-3' and reverse, 5'-CTCAAGCTCATCTA ATCGTCCTG-3'. The relative gene expression was quantified using the $2^{-\Delta \Delta \mathrm{Cq}}$ method (30).

Western blot assay. Radioimmunoprecipitation assay lysis buffer (Beyotime Institute of Biotechnology) was used to collect the proteins from cells, and bicinchoninic acid protein assay was then conducted to quantify the protein concentration. Equal amount of protein (40 $\mu \mathrm{g} /$ lane) was separated by $12 \%$ SDS-PAGE and then transferred to polyvinylidene difluoride membranes. Next, the membranes were blocked with $5 \%$ non-fat milk at room temperature for $1.5 \mathrm{~h}$, incubated with primary antibodies: $\operatorname{Smad} 2$ (1:1,000; cat. no. 5339; Cell Signaling Technology, Inc.), Col I (1:1,000; cat. no. ab34710; Abcam), Col III (1:1,000; cat. no. Ab7778; Abcam) and $\beta$-actin (1:1,000; cat. no. 4970; Cell Signaling Technology, Inc.) at $4^{\circ} \mathrm{C}$ overnight. Subsequently, membranes were incubated with anti-rabbit horseradish peroxidase-conjugated immunoglobulin G secondary antibody (cat. no. 7074; 1:2,000; Cell Signaling Technology, Inc.) at room temperature for $2 \mathrm{~h}$. At the end of the experiment, an enhanced chemiluminescence detection system (Applygen Technologies, Inc., Beijing, China) was used to observe the protein bands. For densitometry detection, analysis with ImageJ 1.38X software (National Institutes of Health, Bethesda, MD, USA) was performed.

Dual-luciferase reporter assay. Bioinformatics analysis using the TargetScanHuman 7.2 tool (www.targetscan.org/vert_72) was performed to predict the target genes of miR-18a-5p. The results revealed the binding sites between miR-18a-5p and the 3'-UTR of Smad2. Next, in order to confirm these binding sites, dual-luciferase reporter assay was conducted (31). Briefly, the Smad2-WT vector with the wild-type 3'-UTR of Smad2 mRNA and the Smad2-MUT vector with the mutated 3'-UTR of Smad 2 mRNA were constructed with the dual-luciferase 

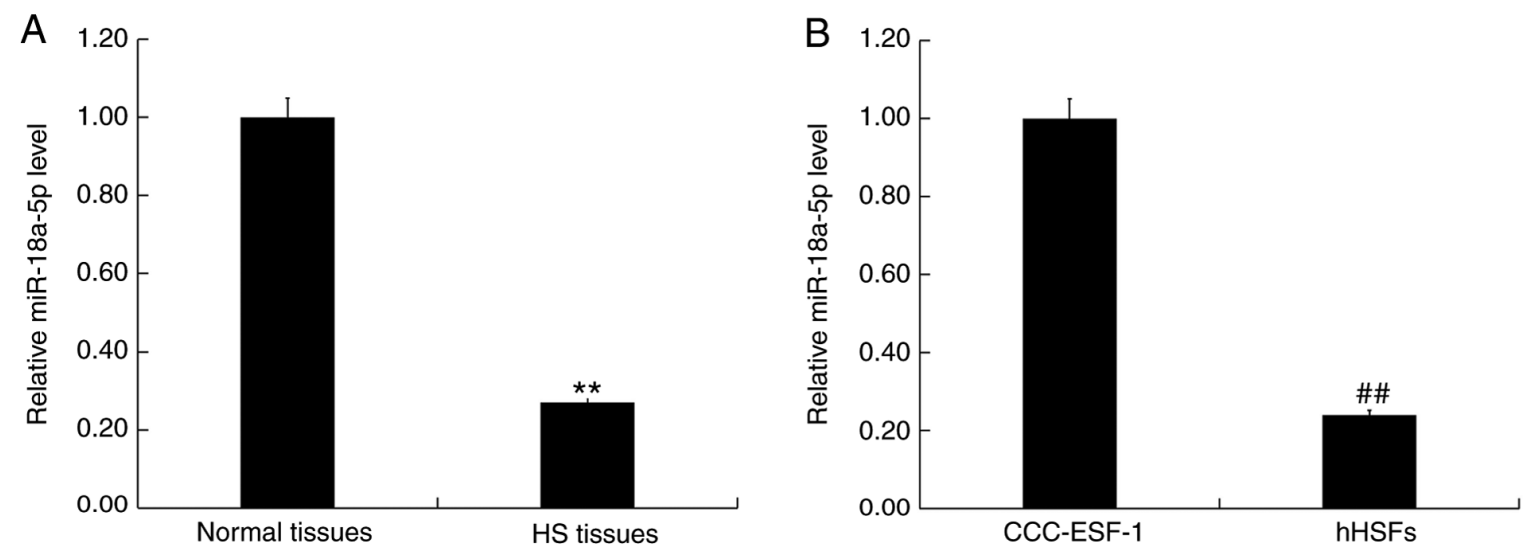

Figure 1. miR-18a-5p expression was downregulated in HS tissues and cells. The relative miR-18a-5p expression was determined in (A) HS and paired normal tissues, and (B) normal CCC-ESF-1 cells and hHSFs. Data are presented as the mean \pm standard deviation. ${ }^{* *} \mathrm{P}<0.01$ vs. normal tissues; ${ }^{\# \#} \mathrm{P}<0.01$ vs. CCC-ESF-1 cells. miR, microRNA; HS, hypertrophic scar; hHSFs, human HS fibroblasts.

reporter vector pmiR-RB-REPORT ${ }^{\mathrm{TM}}$ (Guangzhou RiboBio Co., Ltd.). Subsequently, hHSFs were co-transfected with miR-18a-5p mimic or mimic control, and with Smad2-WT or Smad2-MUT using Lipofectamine ${ }^{\circledR} 3000$ (Invitrogen; Thermo Fisher Scientific, Inc.), according to the manufacturer's protocol. At $48 \mathrm{~h}$ after cell transfection, the relative luciferase activity was measured using a dual-luciferase reporter assay system (Promega Corporation) following the manufacturer's protocols. Luciferase activity was normalized to the Renilla luciferase activity.

MTT assay. To determine cell proliferation, an MTT assay was performed. Briefly, hHSFs were seeded into 96 -well plates ( $1 \times 10^{4}$ cells per well), and then transfected with miR-18a-5p inhibitor, inhibitor control, miR-18a-5p mimic, mimic control or miR-18a-5p mimic + Smad2-plasmid for $48 \mathrm{~h}$. Next, $20 \mu \mathrm{l}$ MTT (5 mg/ml; Sigma-Aldrich; Merck KGaA) was added to each well and cultured at $37^{\circ} \mathrm{C}$ with $5 \% \mathrm{CO}_{2}$ for a further $4 \mathrm{~h}$. The optical density value at $490 \mathrm{~nm}$ was detected using a microplate reader. Experiments were repeated three times.

Flow cytometry assay. hHSFs were collected in the logarithmic growth phase and inoculated into 6-well plates at $1 \times 10^{5}$ cells/well. hHSFs were then transfected with miR-18a-5p inhibitor, inhibitor control, miR-18a-5p mimic, mimic control or miR-18a-5p mimic + Smad2-plasmid for $48 \mathrm{~h}$. Then, cell apoptosis was assessed using the Annexin V-FITC Apoptosis Detection kit (cat.no.70-AP101-100; MultiSciences, Hangzhou, China) and a flow cytometer (BD Biosciences, Franklin Lakes, NJ, USA), according to the manufacturer's protocol. The data were then analyzed using WinMDI software (version 2.5; www.cyto.purdue.edu/flowcyt/software/Catalog.htm).

Statistical analysis. Data are expressed as the mean \pm standard deviation of experiments conducted at least in triplicate. The SPSS software, version 18.0 (IBM Corp., Armonk, NY, USA) was used to perform statistical analysis. Comparisons between groups were assessed by Student's t-test, or by one-way analysis of variance and subsequent Tukey's post-hoc test. $\mathrm{P}<0.05$ was considered to denote a statistically significant difference.
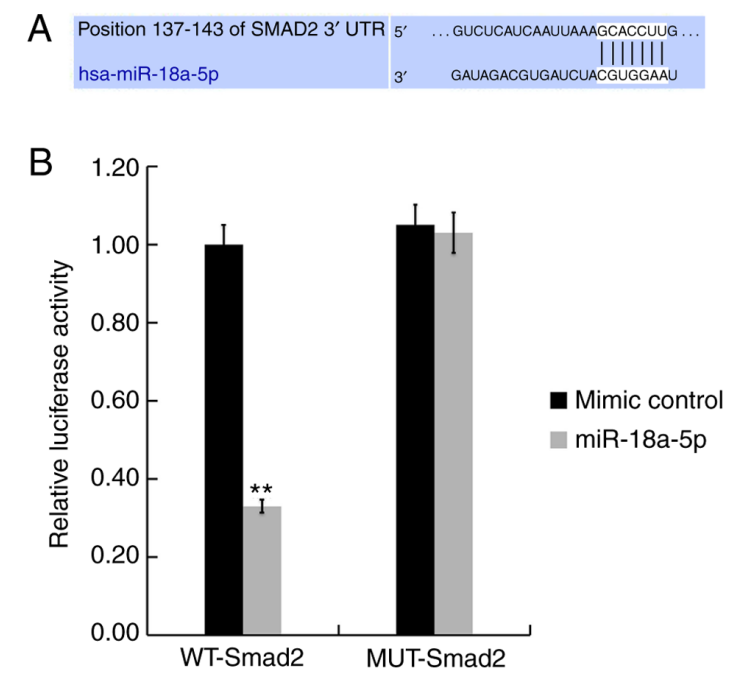

Figure 2. Smad2 is a target gene of miR-18a-5p. (A) TargetScan was used to predict the interaction between miR-18a-5p and the 3'-UTR of Smad2. (B) Luciferase activity was measured by conducting a dual-luciferase reporter assay. Data are presented as the mean \pm standard deviation. ${ }^{* *} \mathrm{P}<0.01$ vs. mimic control. miR, microRNA; WT, wild-type; MUT, mutant; UTR, untranslated region.

\section{Results}

miR-18a-5p is downregulated in HS tissues and hHSFs. In order to detect the level of miR-18a-5p in HS tissues and normal skin tissues, as well as in the human embryonic skin fibroblasts CCC-ESF-1 and hHSFs, RT-qPCR analysis was performed. The results demonstrated that, compared with the normal skin tissues, the level of miR-18a-5p was significantly downregulated in HS tissues (Fig. 1A). Furthermore, compared with the normal fibroblasts CCC-ESF-1, the level of miR-18a-5p in hHSFs was significantly decreased (Fig. 1B).

Smad2 is a target of miR-18a-5p. TargetScanHuman 7.2 was used to conduct bioinformatics analysis, and the binding sites between miR-18a-5p and the 3'-UTR of Smad2 were identified (Fig. 2A). Next, the findings of the dual-luciferase reporter 

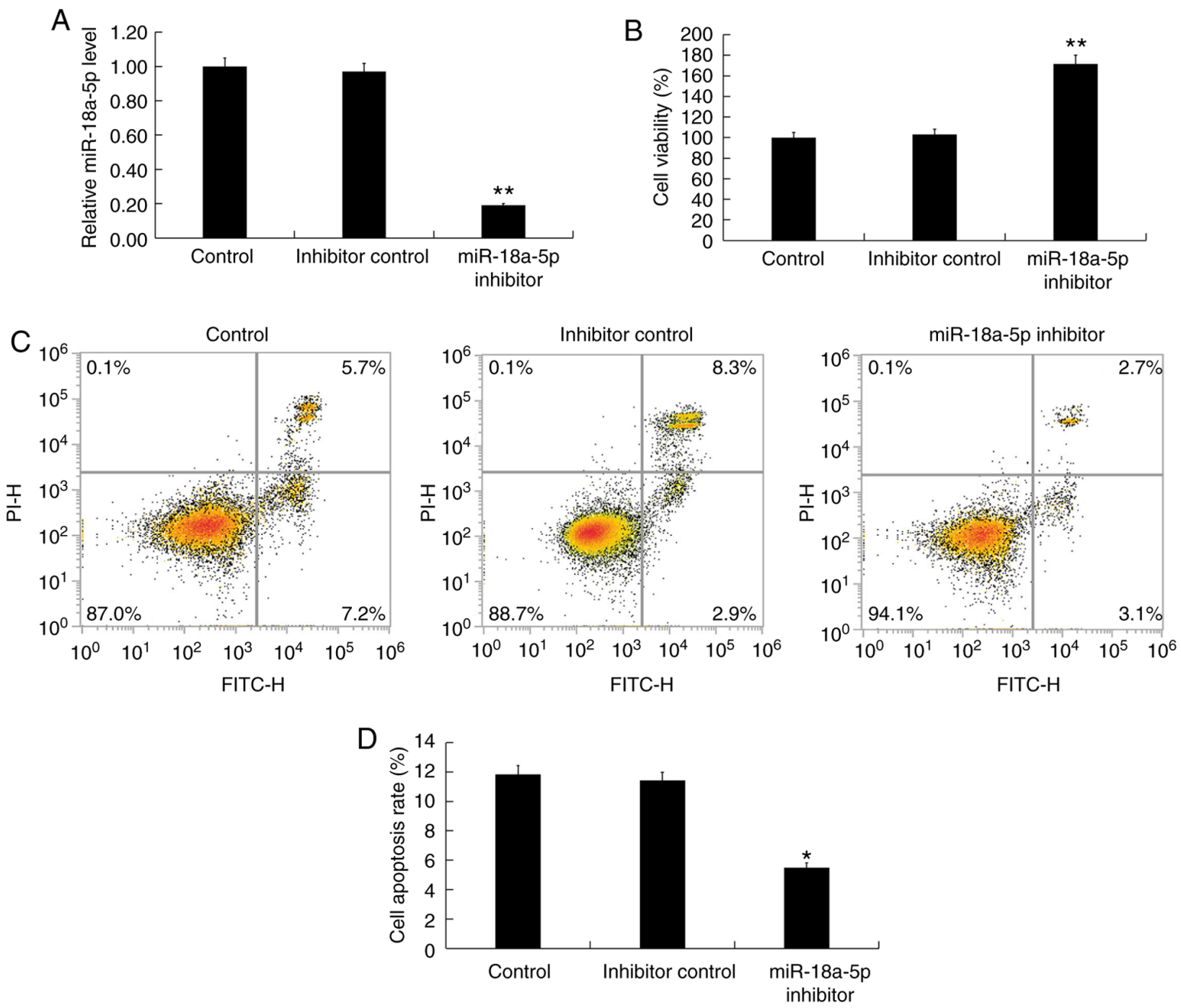

Figure 3. Effect of miR-18a-5p inhibitor on hHSFs. The hHSFs were transfected with inhibitor control or miR-18a-5p inhibitor for $48 \mathrm{~h}$. (A) Reverse transcription-quantitative polymerase chain reaction was used to detect miR-18a-5p level in hHSFs. (B) MTT assay was performed to detect cell viability. (C) Flow cytometry was used to detect cell apoptosis, and (D) the cell apoptosis rate was determined. Data are presented as the mean \pm standard deviation. ${ }^{*} \mathrm{P}<0.05$ and ${ }^{* *} \mathrm{P}<0.01$ vs. control group. miR, microRNA; hHSFs, human hypertrophic scar fibroblasts.

assay indicated that, compared with the mimic control group, miR-18a-5p mimic transfection significantly decreased the luciferase activity of hHSFs co-transfected with Smad2-WT. By contrast, no significant difference was observed in cells co-transfected with Smad2-MUT and miR-18a-5p mimic or mimic control (Fig. 2B). These results indicated that miR-18a-5p directly targets Smad2.

miR-18a-5p inhibition promotes proliferation, inhibits apoptosis and enhances extracellular matrix deposition in hHSFs. Subsequently, in order to investigate the effect of miR-18a-5p downregulation on hHSFs, the hHSFs were transfected with inhibitor control or miR-18a-5p inhibitor for $48 \mathrm{~h}$. The RT-qPCR results indicated that transfection with miR-18a-5p inhibitor significantly decreased the expression of miR-18a-5p in hHSFs, compared with the untransfected control and inhibitor control groups (Fig. 3A). Next, it was observed that, compared with the control groups, miR-18a-5p inhibitor significantly promoted cell proliferation (Fig. 3B), decreased cell apoptosis (Fig. 3C and D), and enhanced the protein (Fig. 4A-D) and mRNA (Fig. 4E-G) expression levels of Smad2 (Fig. 4A, B and E), Col I (Fig. 4A, C and F), and Col III (Fig. 4A, D and G) in hHSFs. These findings suggest that miR-18a-5p inhibitor could enhance extracellular matrix deposition by hHSFs.

miR-18a-5p upregulation inhibits proliferation, induces apoptosis and represses extracellular matrix deposition in hHSFs. Finally, the effect of miR-18a-5p upregulation on hHSFs was investigated, and hHSFs were transfected with miR-18a-5p mimic, mimic control or miR-18a-5p mimic + Smad2-plasmid for $48 \mathrm{~h}$. In addition, in order to confirm the transfection efficiency of Smad2-plasmid, hHSFs were also transfected with control-plasmid or Smad2-plasmid for $48 \mathrm{~h}$. The results confirmed that miR-18a-5p mimic transfection significantly increased the level of miR-18a-5p in hHSFs (Fig. 5A), while Smad2-plasmid transfection significantly enhanced the mRNA level of Smad2 in hHSFs (Fig. 5B). Further analysis indicated that compared with the control groups, miR-18a-5p mimic transfection significantly 
A
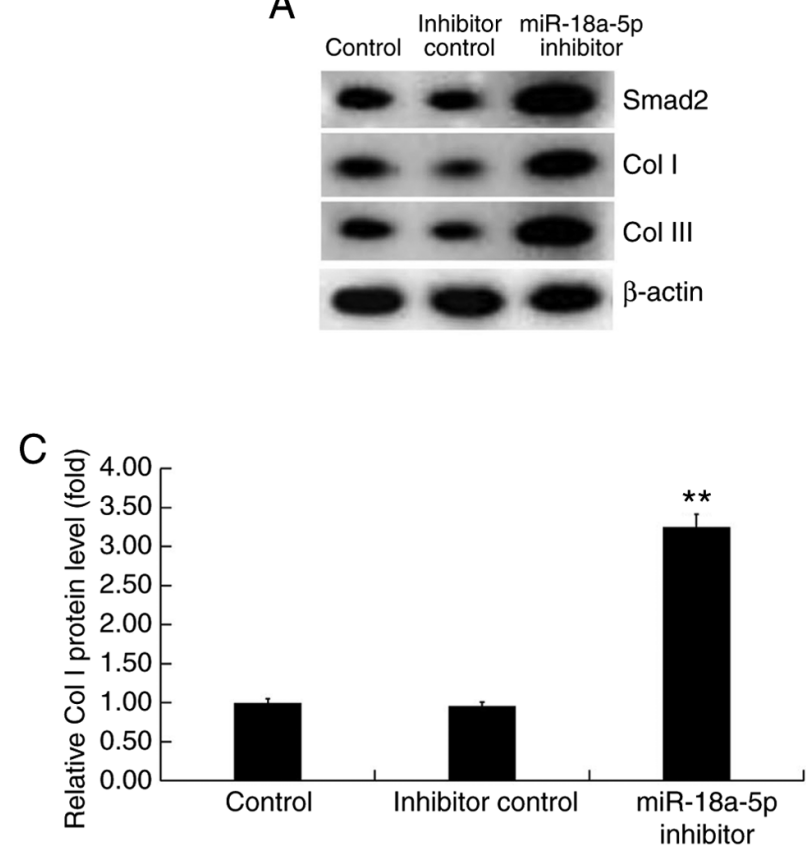

$\mathrm{E}$

$$
\text { क }
$$

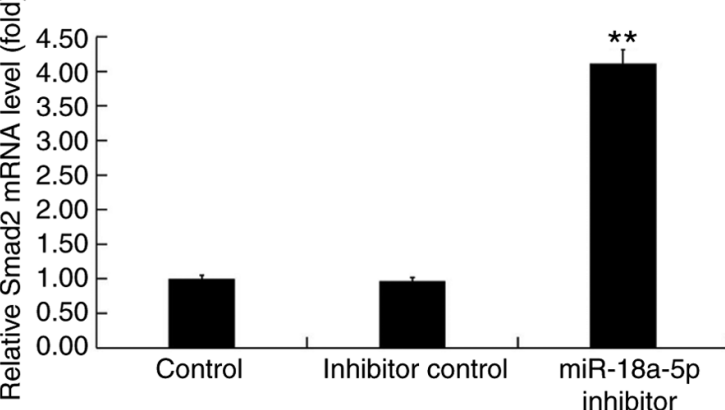

G

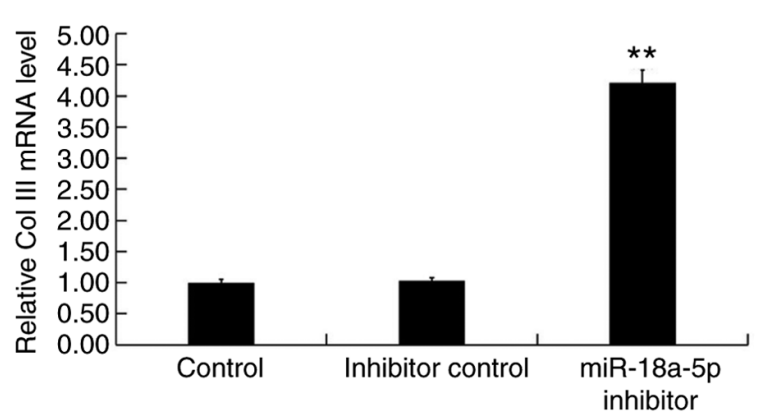

$\mathrm{B}$ 응
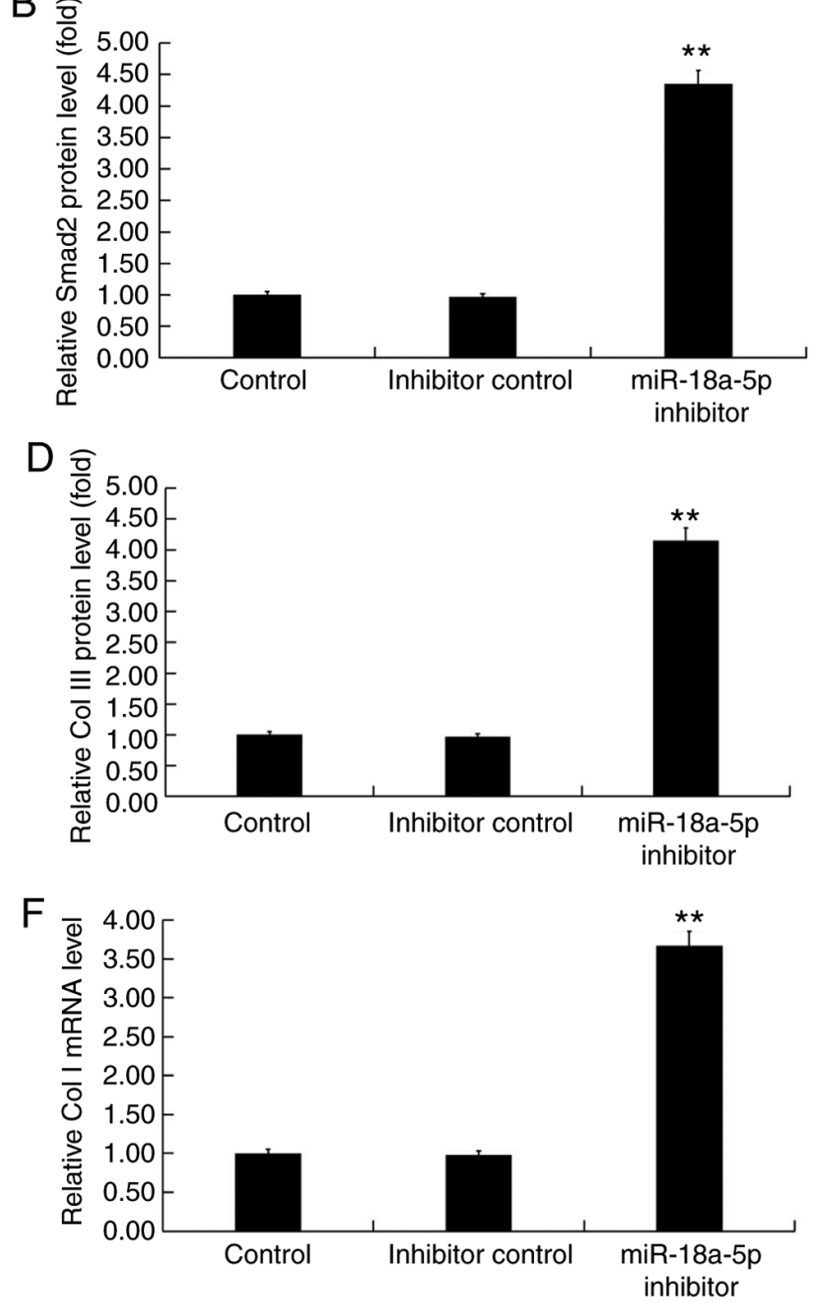

Figure 4. Effect of miR-18a-5p inhibitor on Smad2, Col I and Col III expression levels in hHSFs. The hHSFs were transfected with inhibitor control or miR-18a-5p inhibitor for 48 h. (A) Western blot assay was used to measure the protein levels of Smad2, Col I and Col III in hHSFs. (B) Smad2, (C) Col I and (D) Col III relative protein levels in hHSFs were calculated and are presented as the fold of the control levels. (E) Smad2, (F) Col I and (G) Col III relative mRNA expression levels in hHSFs were measured by reverse transcription-quantitative polymerase chain reaction. Data are presented as the mean \pm standard deviation. ${ }^{* *} \mathrm{P}<0.01$ vs. control group. miR, microRNA; hHSFs, human hypertrophic scar fibroblasts; Col, Collagen.

inhibited the proliferation (Fig. 5C) and increased the apoptosis (Fig. 5D and E) of hHSFs, while it markedly reduced the protein (Fig. 6A-D) and mRNA (Fig. 6E-G) expression levels of Smad2 (Fig. 6A, B and E), Col I (Fig. 6A, C and F), and Col III (Fig. 6A, D and G) in hHSFs. Notably, upon co-transfection of cells with miR-18a-5p mimic and Smad2-plasmid, all the effects of miR-18a-5p mimic on hHSFs were significantly reversed by the Smad2 overexpression (Figs. 5C-E and 6). These findings suggest that miR-18a-5p mimic can reduce extracellular matrix deposition by hHSFs.

\section{Discussion}

In the present study, miR-18a-5p was demonstrated to be significantly downregulated in HS tissues and hHSFs. In addition, the results revealed that $\mathrm{Smad} 2$ is a target of miR-18a-5p, and that it was upregulated in HS tissues and hHSFs. Further analysis indicated that miR-18a-5p downregulation significantly promoted cell proliferation and decreased cell apoptosis, as well as enhanced the expression levels of Smad2, Col I and Col III in hHSFs. By contrast, miR-18a-5p upregulation 

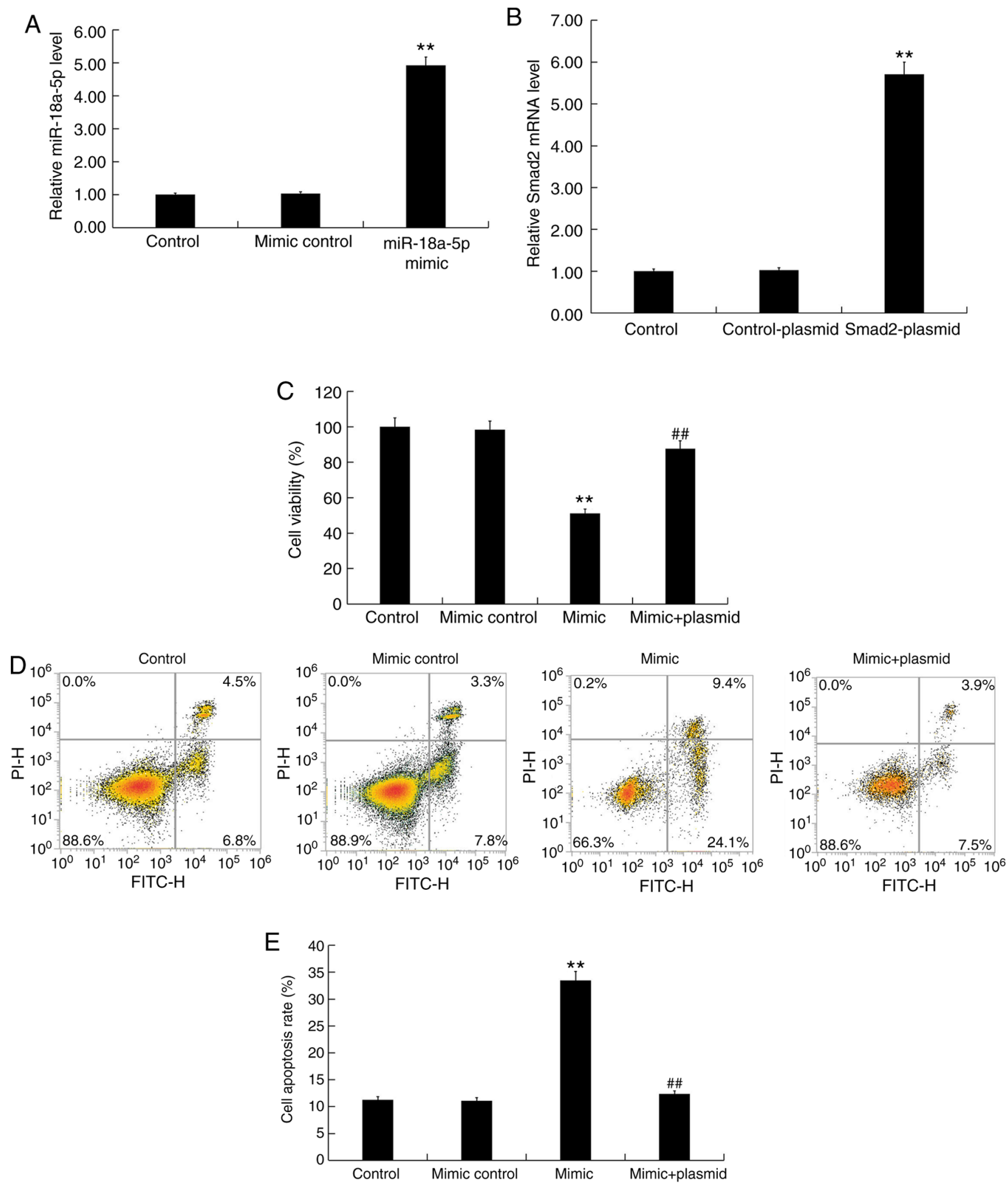

Figure 5. Effect of miR-18a-5p mimic on hHSFs. (A) hHSFs were transfected with mimic control or miR-18a-5p mimic for $48 \mathrm{~h}$, and then RT-qPCR was used to detect the miR-18a-5p level. (B) hHSFs were transfected with control-plasmid or Smad2-plasmid for $48 \mathrm{~h}$, and then RT-qPCR was used to detect Smad2 mRNA level. (C) hHSFs were transfected with mimic control, miR-18a-5p mimic or miR-18a-5p mimic + Smad2-plasmid for $48 \mathrm{~h}$, and then MTT was used to detect cell viability. (D) Flow cytometry graphs and (E) cell apoptosis rate in hHSFs transfected with mimic control, miR-18a-5p mimic or miR-18a-5p mimic + Smad2-plasmid for $48 \mathrm{~h}$. Data are presented as the mean \pm standard deviation. ${ }^{* *} \mathrm{P}<0.01$ vs. control group; ${ }^{\# \prime} \mathrm{P}<0.01$ vs. mimic group. miR, microRNA; hHSFs, human hypertrophic scar fibroblasts; RT-qPCR, reverse transcription-quantitative polymerase chain reaction.

significantly inhibited hHSF proliferation, increased cell apoptosis, and reduced the expression levels of Smad2, Col I and Col III in hHSFs. All the effects of miR-18a-5p upregulation on hHSFs were significantly reversed by Smad2 overexpression. Taken together, the data suggested that the
miR-18a-5p/Smad2 axis may be a potential therapeutic target for HS treatment.

HS formation does not only cause cosmetic disfigurement of the human body, but also leads to discomfort, such as pain and itching, and even serious deformity and loss of function, 

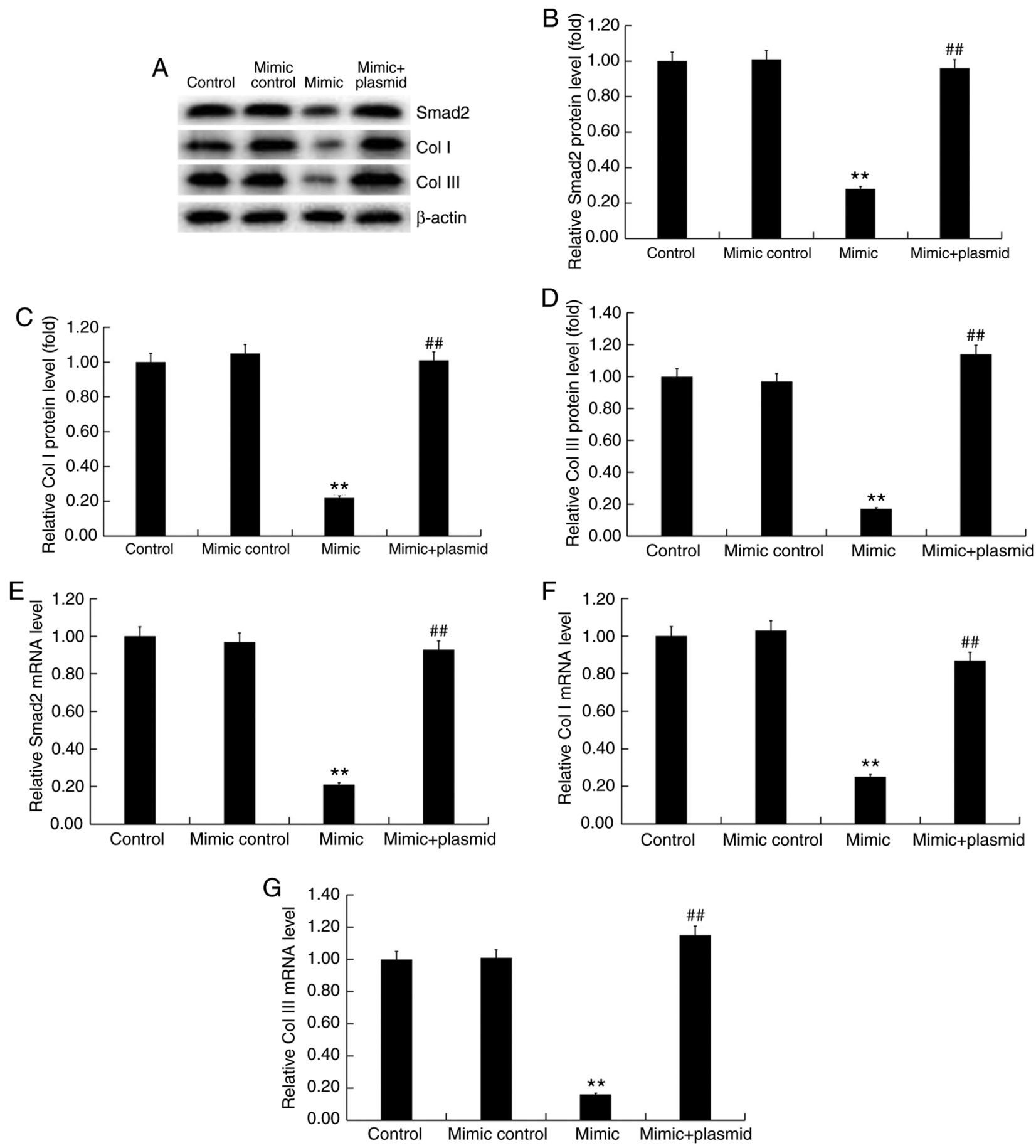

Figure 6. Effect of miR-18a-5p mimic on Smad2, Col I and Col III in hHSFs. The hHSFs were transfected with mimic control, miR-18a-5p mimic, or miR-18a-5p mimic + Smad2-plasmid for $48 \mathrm{~h}$. (A) Western blot assay was performed to measure the protein levels of Smad2, Col I and Col III in hHSFs. (B) Smad2, (C) Col I and (D) Col III relative protein levels in hHSFs were calculated and are presented as the fold of the control levels. (E) Smad2, (F) Col I and (G) Col III mRNA levels in hHSFs were measured by reverse transcription-quantitative polymerase chain reaction. Data are presented as the mean \pm standard deviation. ${ }^{* *} \mathrm{P}<0.01$ vs. control group; ${ }^{\# \#} \mathrm{P}<0.01$ vs. mimic group. miR, microRNA; hHSFs, human hypertrophic scar fibroblasts; Col, Collagen.

affecting the patient's quality of life (29,30,32-34). Therefore, the formation mechanism and treatment methods of HSs have received increasing attention, and methods for effectively eliminating scars have great clinical significance. Current research has confirmed the important role of miRNAs in the development of skin wound repair and skin diseases $(35,36)$, while numerous studies have also reported the important involvement of miRNAs in HS formation (14-17). miR-18a-5p, which has been well studied in cancer, serves a critical role in the regulation of cell growth (18-25). As fibroblast hyperplasia and extracellular matrix deposition are the main features of HS formation $(28,29)$, it was hypothesized in the present study that miR-18a-5p may also serve an important role in the formation of HS.

Initially, the expression of miR-18a-5p in HS and normal skin tissues was measured, and the results indicated that 
miR-18a-5p was significantly downregulated in HS tissues. However, it is worth noting that the age of patients who participated in the current study ranged between 27 and 51 years. Since age affects the characteristics of scar tissue and the quality of life of patients $(37,38)$, the wide age range of patients enrolled in the present study was a limitation of the study. The level of miR-18a-5p in the human embryonic skin fibroblasts CCC-ESF-1 and hHSFs was also detected in the current study using RT-qPCR, and the results indicated that miR-18a-5p was significantly downregulated in hHSFs. Subsequently, Smad2 was identified to be a target of miR-18a-5p. Consistent with the findings of a previous study (39), it was observed herein that Smad2 was significantly upregulated in HS tissues. An earlier study reported that silencing of Smad 2 gene expression was able to inhibit the TGF- $\beta$ signaling pathway and thus reduce HS formation (40). The Smad2/3-TGF- $\beta 1$ signaling pathway serves a key role in the regulation of target gene transcription, matrix protein production (including fibronectin and collagen) and extracellular matrix synthesis and degradation $(41,42)$.

A previous study demonstrated that miR-486-5p inhibited the proliferation, induced apoptosis and G1/S phase arrest in hHSFs by targeting Smad2 (43). In the present study, the effect of miR-18a-5p on the proliferation of hHSFs was investigated. Downregulation of miR-18a-5p in hHSFs significantly promoted the proliferation and inhibited the apoptosis of hHSFs, whereas miR-18a-5p upregulation inhibited the proliferation and increased the apoptosis of hHSFs. All the effects of miR-18a-5p upregulation on the proliferation and apoptosis of hHSFs were significantly reversed by Smad 2 overexpression. Conversely, previous studies have indicated that miR-18a-5p promoted the cell proliferation in certain epithelial cells $(18,25)$, while another study has reported that Smad2 suppressed the proliferation of epithelial cells. These contradictory results may be due to the different functions of miRNAs/genes in different microenvironments, and may also be caused by the varying sensitivity of different genes to different signaling pathways under specific conditions. For instance, miR-18a-5p may inhibit the endothelial-mesenchymal transition and cardiac fibrosis through repressing Notch2 pathway (26). In addition, miR-18a-5p induced intrinsic keratinocyte apoptosis in patients with toxic epidermal necrolysis through downregulating BCL2L10 expression (44). miR-18a-5p was also able to promote MG-63 osteosarcoma cell invasion and migration by directly regulating the expression of interferon regulatory factor 2 (18). However, the specific mechanisms of the different roles of $\mathrm{miR}-18 \mathrm{a}-5 \mathrm{p} / \mathrm{Smad} 2$ in epithelial cells and fibroblasts require further in-depth research.

Collagen deposition caused by collagen metabolism disorder is one of the main pathological bases of HS. Collagen is the main component of extracellular matrix and is mainly secreted by skin fibroblasts. Increased expression of Col I and Col III has been detected in the skin scar tissues following trauma (45). In the present study, the effect of miR-18a-5p on extracellular matrix production was also explored in hHSFs. As expected, the data demonstrated that miR-18a-5p downregulation significantly enhanced the expression levels of Col I and Col III in hHSFs, while miR-18a-5p upregulation resulted in the opposite effect. It is worth mentioning that the effects of miR-18a-5p upregulation on Col I and Col III expression in hHSFs were significantly reversed by Smad 2 overexpression.
However, extracellular matrix deposition was only investigated by western blot assay in the present study, whereas it would be more appropriate to characterize ECM deposition using immunochemistry or immunofluorescence, which is thus a limitation of the present study.

In conclusion, to be best of our knowledge, the present study is the first to reveal that miR-18a-5p is downregulated in HS, and that its upregulation inhibited hHSF proliferation, promoted apoptosis, and reduced Col I and Col III expression levels in hHSFs by targeting Smad2. Therefore, miR-18a-5p may serve as a novel target for the diagnosis and treatment of HS. However, the present study is only a preliminary investigation of the role of miR-18a-5p in HSs. In order to verify the study conclusions, more in-depth research is necessary. For example, the sample size examined was small, and thus the expression of miR-18a-5p in larger HS tissue samples should be analyzed. Furthermore, the correlation between the expression of miR-18a-5p and the clinical features of HS patients, as well as the expression and role of Smad2 in HS, should be further investigated. Besides, the specific mechanism of the positive regulation of Col I and Col III expression levels by Smad 2 observed in the present study needs further exploration. Finally, in vivo studies should be performed to confirm these findings. These issues will be addressed in future studies.

\section{Acknowledgements}

Not applicable.

\section{Funding}

No funding was received.

\section{Availability of data and materials}

The datasets used and/or analyzed during the current study are available from the corresponding author on reasonable request.

\section{Authors' contributions}

TSL contributed to the study design, data collection, statistical analysis, data interpretation and manuscript preparation. YGW and DDL contributed to data collection and data interpretation. LDZ contributed to statistical analysis and literature search. All authors read and approved the final manuscript.

\section{Ethics approval and consent to participate}

Written informed consent was obtained from each patient enrolled in the present study, and the study was approved by Human Ethical Committee of Peking University Shenzhen Hospital (Guangdong, China).

\section{Patient consent for publication}

Not applicable.

\section{Competing interests}

The authors declare that they have no competing interests. 


\section{References}

1. Tyack ZF, Pegg S and Ziviani J: Postburn dyspigmentation: Its assessment, management, and relationship to scarring-a review of the literature. J Burn Care Rehabil 18: 435-440, 1997.

2. Atiyeh BS, El Khatib AM and Dibo SA: Pressure garment therapy (PGT) of burn scars: Evidence-based efficacy. Ann Burns Fire Disasters 26: 205-212, 2013.

3. Gauglitz GG, Korting HC, Pavicic T, Ruzicka T and Jeschke MG Hypertrophic scarring and keloids: Pathomechanisms and current and emerging treatment strategies. Mol Med 17: 113-125, 2011.

4. Younai S, Nichter LS, Wellisz T, Reinisch J, Nimni ME and Tuan TL: Modulation of collagen synthesis by transforming growth factor-beta in keloid and hypertrophic scar fibroblasts. Ann Plast Surg 33: 148-154, 1994.

5. Aarabi S, Bhatt KA, Shi Y, Paterno J, Chang EI, Loh SA, Holmes JW, Longaker MT, Yee H and Gurtner GC: Mechanical load initiates hypertrophic scar formation through decreased cellular apoptosis. FASEB J 21: 3250-3261, 2007.

6. van der Veer WM, Bloemen MC, Ulrich MM, Molema G, van Zuijlen PP, Middelkoop E and Niessen FB: Potential cellular and molecular causes of hypertrophic scar formation. Burns 35 : 15-29, 2009.

7. Atiyeh BS: Nonsurgical management of hypertrophic scars: Evidence-based therapies, standard practices, and emerging methods. Aesthetic Plast Surg 31: 468-492; Discussion 493-494, 2007.

8. Zuccaro J, Ziolkowski N and Fish J: A systematic review of the effectiveness of laser therapy for hypertrophic burn scars. Clin Plast Surg 44: 767-779, 2017.

9. Hammond SM: An overview of microRNAs. Adv Drug Deliv Rev 87: 3-14, 2015.

10. Bartel DP: MicroRNAs: Genomics, biogenesis, mechanism, and function. Cell 116: 281-297, 2004.

11. Mo YY: MicroRNA regulatory networks and human disease. Cell Mol Life Sci 69: 3529, 2012.

12. Laffont B and Rayner KJ: MicroRNAs in the pathobiology and therapy of atherosclerosis. Can J Cardiol 33: 313-324, 2017.

13. Ambros V: The functions of animal microRNAs. Nature 431 : $350-355,2004$

14. Chen L, Li J, Li Q, Yan H, Zhou B, Gao Y and Li J: Non-coding RNAs: The new insight on hypertrophic scar.J Cell Biochem 118 1965-1968, 2017

15. Li P, He QY and Luo CQ: Overexpression of miR-200b inhibits the cell proliferation and promotes apoptosis of human hypertrophic scar fibroblasts in vitro. J Dermatol 41: 903-911, 2014.

16. Wang X, Zhang Y, Jiang BH, Zhang Q, Zhou RP, Zhang L and Wang C: Study on the role of Hsa-miR-31-5p in hypertrophic scar formation and the mechanism. Exp Cell Res 361: 201-209, 2017.

17. Xiao YY, Fan PJ, Lei SR, Qi M and Yang XH: MiR-138/peroxisome proliferator-activated receptor $\beta$ signaling regulates human hypertrophic scar fbroblast proliferation and movement in vitro J Dermatol 42: 485-495, 2015.

18. Lu C, Peng K, Guo H, Ren X, Hu S, Cai Y, Han Y, Ma L and Xu P: miR-18a-5p promotes cell invasion and migration of osteosarcoma by directly targeting IRF2. Oncol Lett 16: 3150-3156, 2018.

19. Fei D, Zhang X, Liu J, Tan L, Xing J, Zhao D and Zhang Y: Long noncoding RNA FER1L4 suppresses tumorigenesis by regulating the expression of PTEN targeting miR-18a-5p in osteosarcoma. Cell Physiol Biochem 51: 1364-1375, 2018.

20. Liu Q, Yu W, Zhu S, Cheng K, Xu H, Lv Y, Long X, Ma L, Huang J, Sun S and Wang K: Long noncoding RNA GAS5 regulates the proliferation, migration, and invasion of glioma cells by negatively regulating miR-18a-5p. J Cell Physiol 234: 757-768, 2018.

21. Zhang N, Zhang H, Liu Y, Su P, Zhang J, Wang X, Sun M, Chen B, Zhao W, Wang L, et al: SREBP1, targeted by miR-18a-5p, modulates epithelial-mesenchymal transition in breast cancer via forming a co-repressor complex with Snail and HDAC1/2. Cell Death Differ 26: 843-859, 2019.

22. Zhang G, Han G, Zhang X, Yu Q, Li Z, Li Z and Li J: Long non-coding RNA FENDRR reduces prostate cancer malignancy by competitively binding miR-18a-5p with RUNX1. Biomarkers 23: 435-445, 2018

23. Zhang Y, Qian W, Feng F, Cao Q, Li Y, Hou Y, Zhang L and Fan J: Upregulated lncRNA CASC2 may inhibit malignant melanoma development through regulating miR-18a-5p/RUNX1. Oncol Res 27: 371-377, 2019.
24. Zhang W, He W, Gao J, Wang Y, Zang W, Dong Z and Zhao G: RETRACTED: The long noncoding RNA CASC2 inhibits tumorigenesis through modulating the expression of PTEN by targeting miR-18a-5p in esophageal carcinoma. Exp Cell Res 361: 30-38, 2017.

25. Zhou L, Li Z, Pan X, Lai Y, Quan J, Zhao L, Xu J, Xu W, Guan X, $\mathrm{Li} \mathrm{H}$, et al: Identification of miR-18a-5p as an oncogene and prognostic biomarker in RCC. Am J Transl Res 10: 1874-1886, 2018.

26. Geng $\mathrm{H}$ and Guan J: MiR-18a-5p inhibits endothelial-mesenchymal transition and cardiac fibrosis through the Notch2 pathway. Biochem Biophys Res Commun 491: 329-336, 2017.

27. Zhang Q, Ye H, Xiang F, Song LJ, Zhou LL, Cai PC, Zhang JC, Yu F, Shi HZ, Su Y, et al: miR-18a-5p inhibits sub-pleural pulmonary fibrosis by targeting TGF- $\beta$ receptor II. Mol Ther 25: 728-738, 2017.

28. Tredget EE, Nedelec B, Scott PG and Ghahary A: Hypertrophic scars, keloids, and contractures. The cellular and molecular basis for therapy. Surg Clin North Am 77: 701-730, 1997.

29. Syed F, Ahmadi E, Iqbal SA, Singh S, McGrouther DA and Bayat A: Fibroblasts from the growing margin of keloid scars produce higher levels of collagen I and III compared with intralesional and extralesional sites: Clinical implications for lesional site-directed therapy. Br J Dermatol 164: 83-96, 2011.

30. Livak KJ and Schmittgen TD: Analysis of relative gene expression data using real-time quantitative PCR and the 2(-Delta Delta C(T)) method. Methods 25: 402-408, 2001.

31. Lu Q, Guo Z and Qian H: Role of microRNA-150-5p/SRCIN1 axis in the progression of breast cancer. Exp Ther Med 17: 2221-2229, 2019

32. Aarabi S, Longaker MT and Gunner GC: Hypertrophic scar formation following burns and trauma: New approaches to treatment. PLoS Med 4: e234, 2007.

33. Gangemi EN, Gregori D, Berchialla P, Zingarelli E, Cairo M, Bollero D, Ganem J, Capocelli R, Cuccuru F, Cassano P, et al: Epidemiology and risk factors for pathologic scarring after burn wounds. Arch Facial Plast Surg 10: 93-102, 2008.

34. Schneider JC, Holavanahalli R, Helm P, Goldstein R and Kowalske K: Contractures in burn injury: Defining the problem. J Burn Care Res 27: 508-514, 2006.

35. Aberdam D, Candi E, Knight RA and Melino G: miRNAs, 'sternness' and skin. Trends Biochem Sci 33: 583-591, 2008.

36. Botchkareva NV: MicroRNA/mRNA regulatory networks in the control of skin development and regeneration. Cell Cycle 11: 468-474, 2012.

37. Butzelaar L, Ulrich MM, Mink van der Molen AB, Niessen FB and Beelen RH: Currently known risk factors for hypertrophic skin scarring: A review. J Plast Reconstr Aesthet Surg 69: 163-169, 2016.

38. Xiao Y, Sun Y, Zhu B, Wang K, Liang P, Liu W, Fu J, Zheng S, Xiao S and Xia Z: Risk factors for hypertrophic burn scar pain, pruritus, and paresthesia development. Wound Repair Regen 26: 172-181, 2018.

39. Qi J, Liu Y, Hu K, Zhang Y, Wu Y and Zhang X: MicroRNA-26a inhibits hyperplastic scar formation by targeting Smad2. Exp Ther Med 15: 4332-4338, 2018.

40. Yin L, Zhao X, Ji S, He C, Wang G, Tang C, Gu S and Yin C: The use of gene activated matrix to mediate effective Smad2 gene silencing against hypertrophic scar. Biomaterials 35: 2488-2498, 2014.

41. Cao S, Xiao L, Rao JN, Zou T, Liu L, Zhang D, Turner DJ, Gorospe $\mathrm{M}$ and Wang JY: Inhibition of Smurf2 translation by miR-322/503 modulates TGF- $\beta /$ Smad 2 signaling and intestinal epithelial homeostasis. Mol Biol Cell 25: 1234-1243, 2014.

42. Yin K, Yin W, Wang Y, Zhou L, Liu Y, Yang G, Wang J and Lu J: MiR-206 suppresses epithelial mesenchymal transition by targeting TGF- $\beta$ signaling in estrogen receptor positive breast cancer cells. Oncotarget 7: 24537-24548, 2016.

43. Shi Y, Wang L, Yu P, Liu Y and Chen W: MicroRNA-486-5p inhibits the growth of human hypertrophic scar fibroblasts by regulating Smad2 expression. Mol Med Rep 19: 5203-5210, 2019.

44. Ichihara A, Wang Z, Jinnin M, Izuno Y, Shimozono N, Yamane K, Fujisawa A, Moriya C, Fukushima S, Inoue $\mathrm{Y}$ and Ihn $\mathrm{H}$ : Upregulation of miR-18a-5p contributes to epidermal necrolysis in severe drug eruptions. J Allergy Clin Immunol 133: 1065-1074, 2014

45. Zhou R, Zhang Q, Zhang Y, Fu S and Wang C: Aberrant miR-21and miR-200b expression and its pro-fbrotic potential in hypertrophic scars. Exp Cell Res 339: 360-366, 2015.

This work is licensed under a Creative Commons Attribution-NonCommercial-NoDerivatives 4.0 International (CC BY-NC-ND 4.0) License. 